\title{
Analog Neural Network based on Memristor Crossbar Arrays
}

\author{
Hacer A. Yildiz ${ }^{1}$, Mustafa Altun ${ }^{1}$, Ali Dogus Gungordu ${ }^{1}$ and Mircea R. Stan ${ }^{2}$ \\ ${ }^{1}$ Faculty of Electrical\&Electronics Eng., Istanbul Technical University 34469, Maslak, Istanbul, Turkey \\ \{haceryildiz, altunmus, gungordua\}@itu.edu.tr \\ ${ }^{2}$ Dept. of ECE, University of Virginia, Charlottesville, Virginia, USA \\ mircea@virginia.edu
}

\begin{abstract}
In this paper, a new feed forward analog neural network is designed using a memristor based crossbar array architecture. This structure consists of positive and negative polarity connection matrices. In order to show the performance and usefulness of the proposed circuit, it is considered a sample application of iris data recognition. The proposed neural network implementation is approved by the simulation in Cadence design environment using $0.35 \mu \mathrm{m}$ CMOS technology. The results obtained are promising for the implementation of high density neural network.
\end{abstract}

\section{Introduction}

Many investigations on the implementation of analog neural networks field are presented in the literature [1-4]. A neural network is made up of a number of artificial neurons and a huge number of interconnections between them. A typical blockdiagram of a feed-forward neural network is shown in Fig.1 [5].

The main task in realizing high efficient analog neural network is to implement the basic block which operates as a linear weighted summer. This block is also referred to as synaptic interconnection stage.

Multilayer feed forward networks, a widely used neural network architecture, require large number of synaptic interconnections (multipliers). Therefore, careful design of multiplier is critical in achieving compact silicon area, minimizing power consumption and improving input range [6-8]

On the other hand, with the discovery of the memristor (memory element) and its efficient use in crossbar arrays offering dense and regular structures [9-11], it is possible to perform multiplication and addition operations in the analog domain in a very effective way [12-14].

As is known from the literature, the memristor element has a number of advantages, such as low power consumption, nonvolatile storage element, excellent scalability and integration [15]. Therefore, the fact that the memristor can be implemented as an electronic synapse in neural network applications, makes this element extremely attractive.

In this paper we discuss the realization of the feed-forward neural network using memristive crossbar. The circuit is realized in cadence design environment using the macro model of memristor. Proper operation of the neural network is illustrated using iris data as a benchmark.

This work is part of a project that has received funding from the European Union's H2020 research and innovation programme under the Marie Sklodowska-Curie grant agreement \#691178
The paper is planned as follows: In section2, the dynamic model of the memristor element and its function in a crossbar array are briefly explained. Proposed neuron circuit realization with memristor crossbar array is presented in Section 3. The simulation results are given in Section 4. Finally, conclusion part is presented in Section 5.

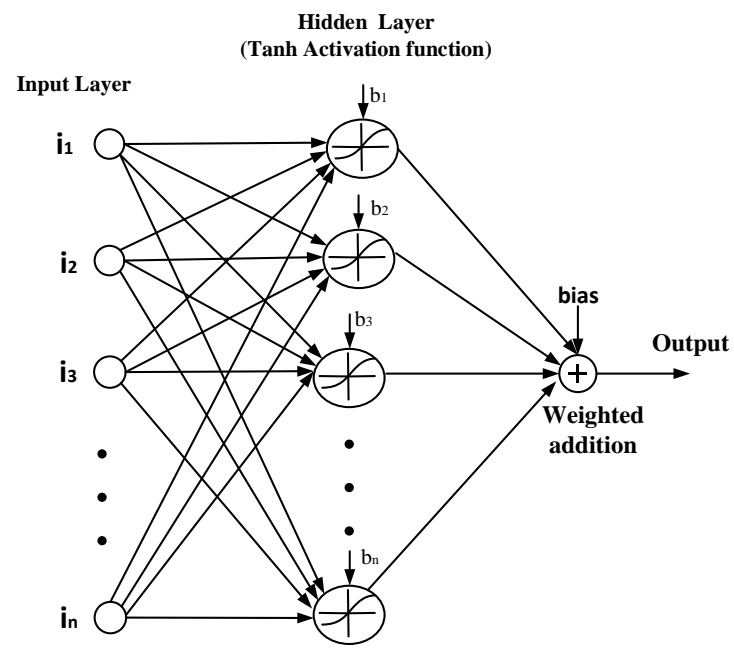

Fig.1. General structure of feed-forward neural network

2. Memristor Device
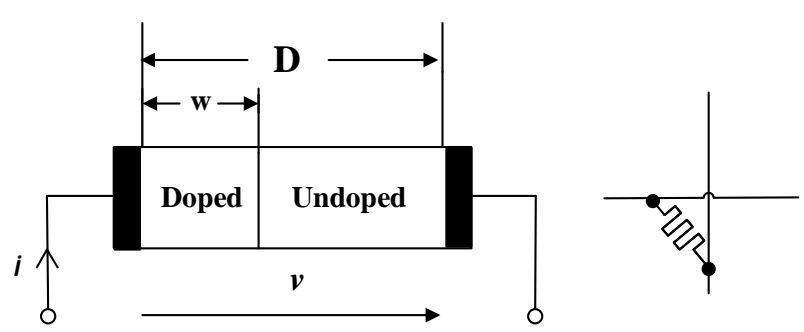

(a)

(b)

Fig.2. (a) Memristor structure, (b) Circuit notation

The memristor is the fourth fundamental element conjectured by Chua in 1971 [16]. The physical memristor device is first introduced by HP Labs 37 years after [17]. The fact that the conceptual Chua's memristor can be realized using HP Lab's nanoscale device inspired the researchers to use this element in the implementations of high density systems, such as non-volatile memory implementations and synapse realizations in neural network circuits. 
The schematic and crossbar circuit symbol of the memristor is shown in Fig. 2. The element has two states, one corresponding to the closed state, where the element displays a low resistance with $\mathrm{w} \cong \mathrm{D},\left(R_{O N}\right)$; while the other corresponds to the open state, where the element shows a high resistance $(\mathrm{w} \cong 0),($ ROFF $)$.

The total resistance $R_{M E M}$ of the memristor can be shown by the following relation:

$$
\begin{gathered}
R_{M E M}(x)=R_{O N} x+R_{O F F}(1-x), \\
\text { where } x=\frac{w}{D} \in(0,1)
\end{gathered}
$$

The relationship between the current and voltage of the memristor can be defined as follows:

$$
v(t)=R_{M E M}(w) i(t)
$$

Depending on RON resistance, passing current and other factors, the speed of movement of the boundary between the doped and undoped regions can be shown as follows:

$$
\frac{d x}{d t}=k i(t) f(x), \quad k=\frac{\mu_{v} R_{O N}}{D^{2}}
$$

where $\mu_{v}$ is called as dopant mobility and the value of $\mu_{v}$ is approximately is $10^{-14} \mathrm{~m}^{2} \mathrm{~s}^{-1} \mathrm{~V}^{-1}$.

Figure. 3 shows device schematic and crossbar circuit notation of a memristor. This circuit can be used to effectively realize the synaptic weights of neural networks. The conductance of each memristor represents a weight. Since memristor is an element with memory, the use of the memristive crossbar adds dynamical characteristics to the synaptic weights. Beside this, the availability of the nanoscale devices allows the implementation of the neural network very effectively in a very small physical area.

Analog input values are represented by symmetrical current or voltage pulses and outputs are obtained as voltage pulses. Thanks to this future, many of the basic arithmetic operation such as summing and multiplication can be realized very effectively in a single step [18].

\section{G}

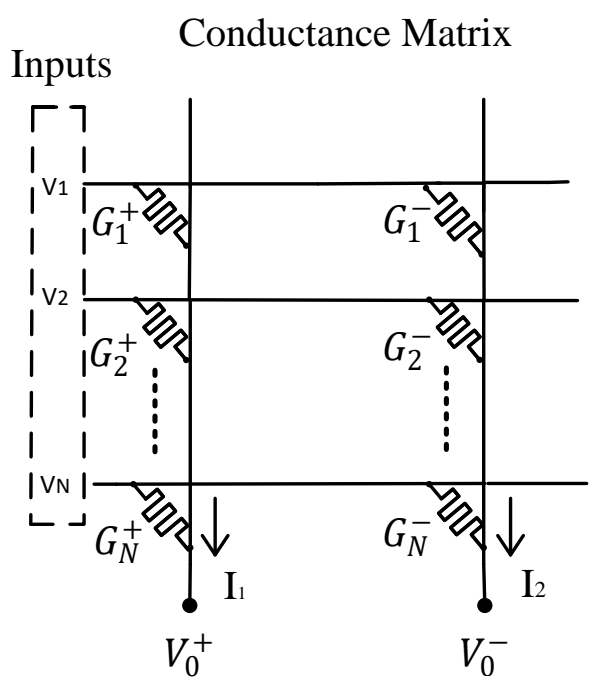

Fig.3. The principle of analog vector-matrix multiplication with memristor array [19].
As it can be seen clearly from Fig.3, the vector multiplication can be naturally processed by directly utilizing the Kirchoff's law. Through Kirchhoff's laws, the applied voltages, $V_{i}$ are multiplied by the conductances $G_{i}^{+}$and $G_{i}^{-}$to give output voltages:

$$
V_{0}^{+}-V_{0}^{-}=\sum \frac{V_{i}\left(G_{i}^{+}-G_{i}^{-}\right)}{G_{0}}
$$

$$
\text { where } G_{0}=G_{1}^{+}+G_{2}^{+} \ldots G_{N}^{+}=G_{1}^{-}+G_{2}^{-} \ldots G_{N}^{-}
$$

\section{Proposed Neuron Circuit Realization with Memristor Crossbar Array}

In this work, the design of neural network using memristor crossbar arrays is presented. In this way, a new memristor-based architecture is realized which suits well high density circuit implementation thanks to the nanoscale realization of memristors. As it can be seeen from the Fig. 4, neural network consists of one hidden layer with tanh type nonlinearity and one linear output neuron.

The MOS transistor at the input layer realize tanh type activation functions as shown in Fig1. The output neuron is a simple linear perceptron, that is why, we use two voltage dividers in order to scale down the signal levels applied to the inputs of the output transistors which ensures the linear operation of these transistors. In addition to, the proposed circuit occupies small area on chip which is very important advantage from IC realization point of view.

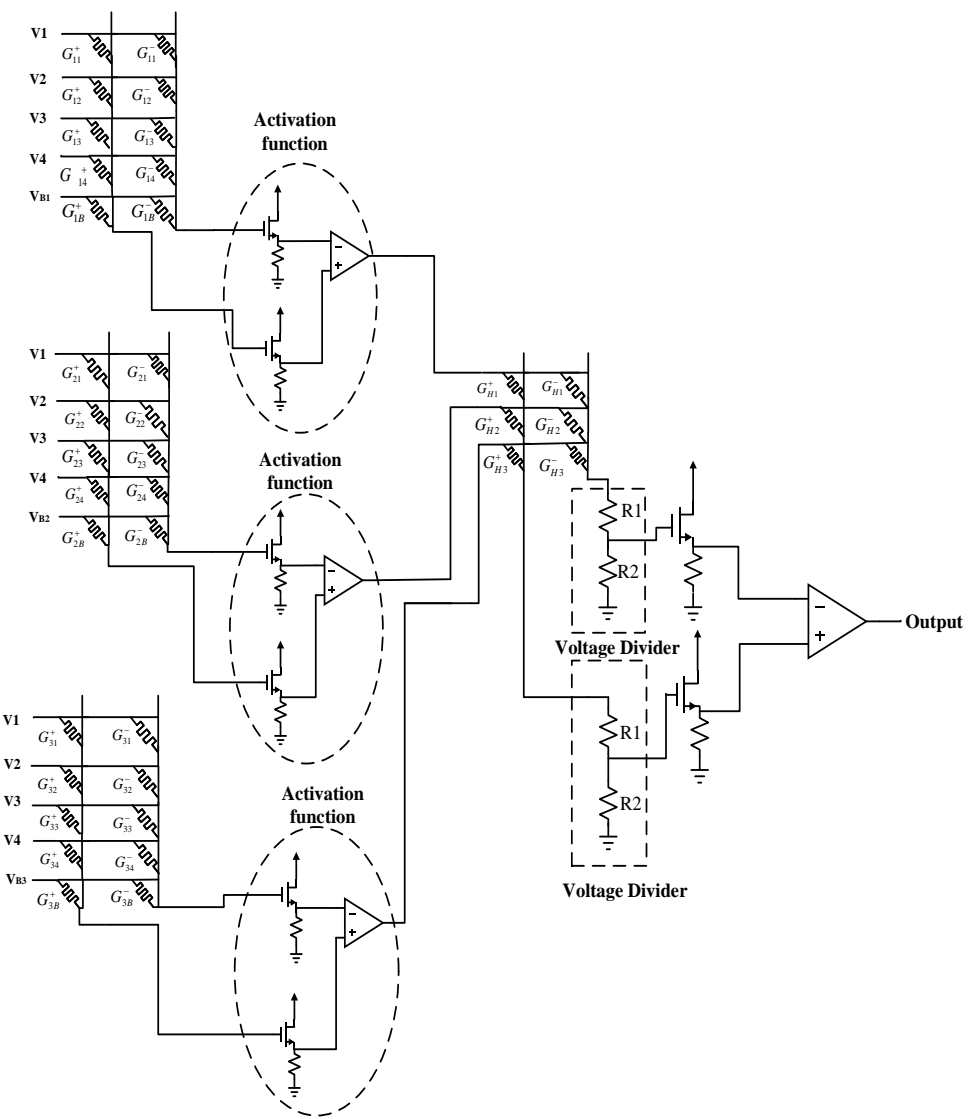

Fig.4. Circuit diagram of the proposed neural network. 
In the simulation of the proposed neural network (Fig.4), the equivalent circuit shown in Fig.5 is used for each memristor element.
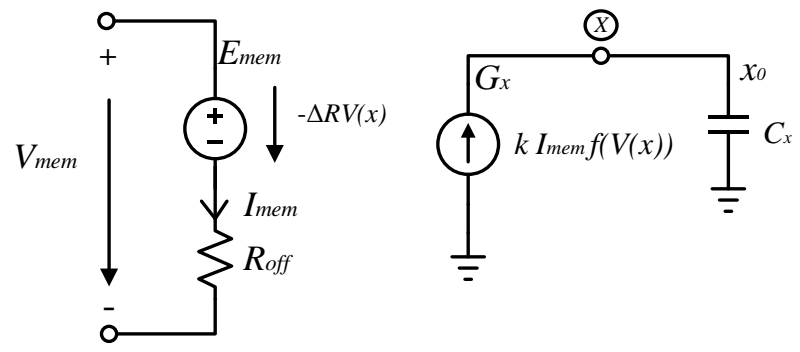

Fig.5. Structure of the SPICE model [20]

The equation shown in (eq.1) can be rearranged as follows:

$$
R_{M E M}(x)=R_{O F F}-x \Delta R, \quad \Delta R=R_{O F F}-R_{O N}
$$

In this model, the initial width of the doped region is modeled by the initial voltage of the capacitor. This initial voltage can be given in terms of the physical quantities as follows:

$$
x_{0}=\frac{R_{O F F}-R_{I N I T}}{\Delta R}
$$

\section{Simulation Results}

In order to verify the feasibility of the proposed neural network with memristive crossbar synapsis, we simulated the proposed circuit in Cadence design environment using 0.35um CMOS technology. The circuit is biased at $\pm 1 \mathrm{~V}$. Baluns are realized using differential pair [21]. In order to realize the memristors in the crossbar, we use the model of Biolek [20]. The memristive weights are obtained by training the network using iris data which is conventionally used in the literature as a benchmark. The parameters of the memristors in neural network in Fig. 4 are trained using backpropagation algorithm set-up on MATLAB environment and these parameters are inserted to the Cadence netlist. The input signals are applied as a pulse with a $10 \%$ duty cycle. The amplitude of the pulses is set according to the iris data.

As it can be seen from Fig.4, there are three memristor crossbar arrays in the input layer and one crossbar array in the output layer.

The waveform of a typical input signal applied to the neural network is given in Fig. 6a. The amplitude of this pulse signal is set to the scaled data corresponding to the sampled iris flower. The signal in Fig. 6a is the first input of the sample shown in Table. 1 .

In order to train the circuit, we have extracted mathematical model of the circuit, that is all memristors are modelled by the differential equation in Eqs. (1-4). The subcircuits consisting of MOS transistors and Baluns (used for converting from differential signal to the single ended signal) are modelled with tanh function. The overall system model thus obtained is inserted to the Simulink Matlab and trained based on iris flower data set [22] using back propagation algorithm.
Table.1 The simulation results of the neural network for three sample inputs.

\begin{tabular}{|l|c|c|c|c|c|c|}
\hline & $\mathrm{V}_{\mathrm{i} 1}$ & $\mathrm{~V}_{\mathrm{i} 2}$ & $\mathrm{~V}_{\mathrm{i} 3}$ & $\mathrm{~V}_{\mathrm{i} 4}$ & $\mathrm{~V}_{0, \text { ideal }}$ & $\mathrm{V}_{0}$ \\
\hline Sample1 & -0.556 & 0.250 & -0.864 & -0.917 & -1.048 & -1.13 \\
\hline Sample2 & -0.111 & -0.167 & 0.390 & 0.417 & 0.727 & 0.8 \\
\hline Sample3 & 0.333 & -0.083 & 0.559 & 0.917 & 1.07 & 1.1 \\
\hline
\end{tabular}

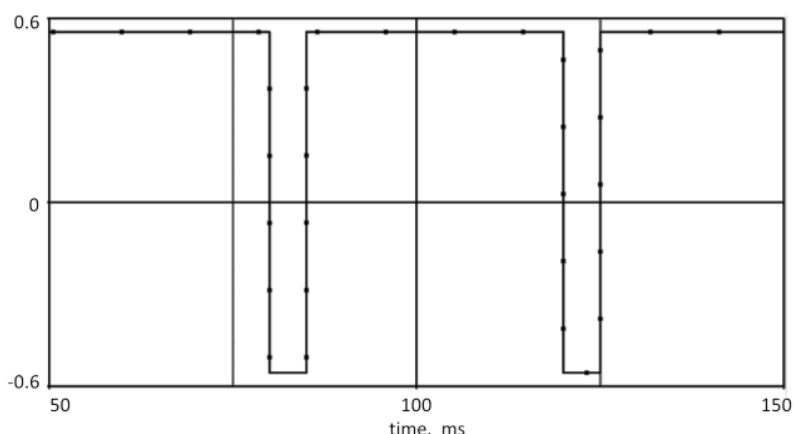

(a)

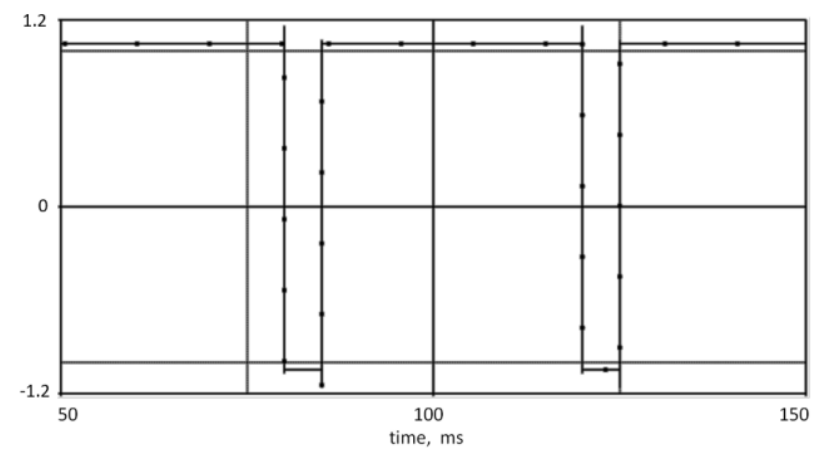

(b)

Fig.6 a) Typical signal shown for $\mathrm{V}_{\mathrm{il}}=-0.556 \mathrm{~V}$ applied to neural network input $b$ ) The corresponding output signal $\left(\mathrm{V}_{\text {out }}=-1.13 \mathrm{~V}\right)$

\section{Conclusions}

In this paper, the design of analog neural network with memristor crossbar arrays is considered. The required activation functions are realized using sub-circuits consisting of Baluns and CMOS transistors. Iris data recognition application is used to test the performance of the proposed neural network. For this purpose, the back propagation algorithm is used in MATLAB environment and then obtained parameters are used in Cadence netlist. Thus, the performance of the proposed circuit is verified for the application of the iris data recognition.

\section{References}

[1] P. Moerland, E. Fiesler, "Neural network adaptations to hardware implementation", IDIAP'97, 1997.

[2] S. Draghici, "Network in analog hardware-design and implementation issues", Int. J. of Neural Systems, 2000, vol.10, no. 1, pp. 19-42.

[3] J. Madrenas, J. Cosp, O. Lucas, E. Alarcon, E. Vidal, E. Villar, G. Bioseg, "A bioinspired VLSI analog system for 
image segmentation", European Symposium on Artificial Neural Networks, 2004, pp. 411-416.

[4] J. Fieres, A. Grübl, S. Philipp, K. Meier, S. Johannes, F. Schürmann, "A platform for parallel operation of VLSI neural networks", BICS 2004.

[5] K.Nakamura, M.Hotta, L.R.Carley, and D.J.Allstot, "An 85 $\mathrm{mW}, 10 \mathrm{~b}, 40 \mathrm{Msample/s}$ CMOS parallel-pipelined ADC"،, IEEE J. Solid-State Circuits, vol. 30, pp. 173-183, 1995.

[6] M. Mirhassani, M. Ahmadi, and G. A. Jullien, "Robust lowsensitivity adaline neuron based on continuous valued number system," J. Analog Integr. Circuits Signal Process. vol. 56, pp. 223-231, Apr. 2008.

[7] H. Djahanshahi, M. Ahmadi, G. A. Jullien, and W. C. Miller, "Sensitivity study and improvements on a nonlinear resistive-type neuron circuit", IEE Proc.-Circuits, Devices Syst., vol. 147, no. 4, pp. 237-242, Aug. 2000.

[8] L. Gatet, H. T. Beteille, and F. Bony, "Comparison between analog and digital neural network implementations for range-finding applications," IEEE Trans. Neural Netw., vol. 20, no. 3, pp. 460-470, Mar.2009.

[9] D. Alexandrescu, M. Altun, L. Anghel, A. Bernasconi, V. Ciriani, L. Frontini and M. Tahoori, "Logic synthesis and testing techniques for switching nano-crossbar arrays", Microprocessors and Microsystems, 54, pp. 14-25, 2017.

[10] O. Tunali, M. Ceylan Morgul and M.Altun, "Defect-tolerant logic synthesis for memristor crossbars with performance evaluation", IEEE Micro, vol. 38 (5), 2018.

[11] M. Ceylan Morgul, et al.,"Integrated synthesis methodology for crossbar arrays", NANOARCH'18, Athens, Greece, 1819 July 2018 ,

[12] M. S. Tarkov, "Mapping neural network computations onto memristor crossbar", SIBCON 2015.

[13] M. Nourazar, V. Rashtchi, A. Azarpeyvand, F. M. Bayat, "Memristor-based approximate matrix multiplier", Analog Integrated Circuits and Signal Processing, , vol. 93, issue 2, pp 363-373, November 2017.

[14] F. M. Bayat, M. Prezioso, B. Chakrabarti, H. Nili, I. Kataeva D. Strukov, "Implementation of multilayer perceptron network with highly uniform passive memristive crossbar circuits", Nature Communications (9), 2331 (2018).

[15] Y. Pershin, and M. Di Ventra, "Experimental demonstration of associative memory with memristive neural networks," in Neural Networks, vol. 23, no. 7, 2010, pp.881-886.

[16] L. O. Chua, "Memristor - the missing circuit element," IEEE Transactions on Circuit Theory, vol. CT-18 (5), pp. 507-519, 1971.

[17] G. S. S. D. R. S. Dmitri B. Strukov and R. S. Williams, "The missing memristor found," Nature, vol. 453, pp. 4, 2008

[18] M. Hu, H. Li, Q. Wu, G. S. Rose, Y. Chen, "Memristor crossbar based hardware realization of BSB recall function", International Joint Conference on Neural Networks, Jun 2012, pp.1-7.

[19] C. Li et al., "Analogue signal and image processing with large memristor crossbars," Nature Electronics, vol 1, pp. 52-59, 2018.

[20] Z. Biolek, D. Biolek, V. Biolkova, "SPICE Model of memristor with nonlinear dopant drif", Radioengineering, vol. 18, no. 2, pp. 210-214, 2009.

[21] S. Natarajan, "Theory and design of linear active networks" McGraw-Hill, 1994.

[22] R. A. Fisher, "The use of multiple measurements in taxonomic problems", Annals of Eugenics. 7 (2): 179-188, 1936. 